\title{
Clinical and diagnostic findings in 20 patients with toxoplasmosis and the Acquired Immune Deficiency Syndrome
}

\author{
R. E. HOLLIMAN \\ PHLS Toxoplasma Reference Laboratory. St George's Hospital, Blackshaw Road, London SW17 OQT
}

\begin{abstract}
Summary. The clinical and diagnostic findings in 20 cases of toxoplasmosis associated with the Acquired Immune Deficiency Syndrome (AIDS) were investigated in a prospective study of patients in south-east England. Individual details were recorded by a standardised questionnaire incorporating an evaluation of initial presentation, radiological findings, therapy and clinical progression. Toxoplasma serology was performed by reference assays and the presence of the parasite in tissue was sought by culture, histological examination and specific DNA detection. Clinical findings at presentation and results of radiological investigation were comparable with previously published experience from the USA. In contrast, sampling of the cerebrospinal fluid was rarely performed and was found to be of little clinical value. Examination of tissue samples was diagnostic when available, but was not performed routinely. Sulphonamide plus pyrimethamine was the treatment of choice in most cases and response to therapy was satisfactory, although a high incidence of toxicity was recorded.
\end{abstract}

\section{Introduction}

Toxoplasmosis is an important cause of morbidity and mortality amongst immunocompromised patients. Organ-transplant recipients, fetuses, cancer patients and persons with the Acquired Immune Deficiency Syndrome (AIDS) are at risk of severe or lifethreatening infection with Toxoplasma gondii. ${ }^{1}$ Clinical experience of congenital toxoplasmosis ${ }^{2}$ and transplant-related disease ${ }^{3}$ has shown variation between different geographical areas. Several studies of toxoplasmosis associated with AIDS have been published in North America ${ }^{4}$ but there are few reports of this condition in the UK. ${ }^{5}$ Consequently an analysis was undertaken of a series of cases of toxoplasma infection in AIDS patients, diagnosed and managed in south-east England.

\section{Patients and methods}

Samples were received from AIDS patients with a provisional diagnosis of toxoplasma infection. Details of age, sex, clinical presentation and diagnostic findings were recorded with a standardised questionnaire. The presence of toxoplasma specific antibody was determined with the dye test (DT) ${ }^{6}$, latex agglutination test (LAT), ${ }^{7}$ IgM double sandwich enzyme-linked immunosorbent assay (DS-ELISA) ${ }^{8}$ and IgM immunosorbent agglutination assay (IS-
AGA). ${ }^{9}$ The presence of toxoplasma specific nucleic acid was detected by a method based upon amplification of the target DNA by the polymerase chain reaction (PCR). ${ }^{10}$ Isolation of the parasite from clinical samples was attempted by inoculation on to human embryonic lung cell lines. ${ }^{11}$ Local regulations did not permit the injection of tissues from AIDS patients into laboratory animals. Follow-up assessment included details of treatment and clinical progression. Cerebral toxoplasmosis was diagnosed on the basis of histological findings or compatible clinical presentation, radiological results and response to specific therapy. ${ }^{12}$

\section{Results}

Twenty cases of toxoplasmosis associated with AIDS were identified. The patients were 19 male homosexuals with an average age of 45 years (range 28-62 years) and a female intravenous drug abuser aged 33 years. All except one black male patient were of Caucasian origin. Detailed clinical findings at presentation were documented in 12 cases; eight patients had acute deterioration of mental state, eight showed focal neurological signs, six complained of persistent headache, two were pyrexial at presentation, and cachexia, vertigo and visual disturbance were each reported once. Computed tomography (CT) studies were done in 12 cases; in 11 of these patients, multiple discrete lesions were identified. Contrast studies were performed on seven patients and ring 
enhancement of the lesions was reported in every case. Cerebral oedema with mass effect was noted in four scans. Results of toxoplasma specific serology are presented in the table.

Toxoplasma specific immunoglobulin was detected by the DT at the time of presentation in all 20 patients; discordant LAT reactions were not recorded. Specific antibody levels associated with cerebral toxoplasmosis were highly variable, ranging from 16 to $32000 \mathrm{IU}$, measured by the DT, and LAT titres were in the range 64-64000. Significantly increasing levels of specific antibody, demonstrated by greater than a four-fold difference in successive DT results, were found in two patients and were associated with a detectable IgM

Table. Serological findings in AIDS patients with cerebral toxoplasmosis

\begin{tabular}{|c|c|c|c|c|c|}
\hline \multirow{2}{*}{$\begin{array}{c}\text { Case } \\
\text { no. }\end{array}$} & \multirow{2}{*}{$\begin{array}{l}\text { Timing* of } \\
\text { investigation } \\
\text { (weeks) }\end{array}$} & \multirow[b]{2}{*}{ DT (IU) } & \multirow{2}{*}{$\begin{array}{c}\text { LAT } \\
\text { (titre) }\end{array}$} & \multicolumn{2}{|c|}{ IgM detected by } \\
\hline & & & & DS-ELISA & ISAGA \\
\hline \multirow[t]{3}{*}{1} & -8 & 62 & 128 & - & - \\
\hline & 0 & 500 & 128 & - & - \\
\hline & +2 & 500 & 128 & - & - \\
\hline \multirow[t]{3}{*}{2} & 0 & 16 & 64 & - & - \\
\hline & +16 & 31 & 64 & - & - \\
\hline & +32 & 7 & 32 & - & - \\
\hline \multirow[t]{3}{*}{3} & 0 & 62 & 1000 & - & - \\
\hline & +2 & 62 & 1000 & - & - \\
\hline & +4 & 31 & 512 & - & - \\
\hline 4 & 0 & 125 & 128 & - & - \\
\hline 5 & 0 & 125 & 256 & - & - \\
\hline \multirow[t]{4}{*}{6} & 0 & 500 & NT & + & + \\
\hline & +4 & 1000 & NT & + & + \\
\hline & +8 & 250 & NT & + & + \\
\hline & +20 & 500 & NT & + & + \\
\hline 7 & 0 & 1000 & 2000 & - & - \\
\hline \multirow[t]{2}{*}{8} & 0 & 62 & 128 & - & - \\
\hline & +12 & 31 & 256 & - & - \\
\hline \multirow[t]{2}{*}{9} & 0 & 500 & 2000 & - & - \\
\hline & +4 & 250 & 1000 & - & - \\
\hline \multirow[t]{4}{*}{10} & 0 & 32 & 128 & + & + \\
\hline & +4 & 500 & 512 & + & + \\
\hline & +6 & 2000 & 1000 & + & + \\
\hline & +8 & 2000 & 512 & + & + \\
\hline 11 & 0 & 2000 & 4000 & + & + \\
\hline \multirow[t]{3}{*}{12} & -3 & 250 & 256 & - & - \\
\hline & 0 & 125 & 256 & - & + \\
\hline & +2 & 125 & 256 & - & + \\
\hline \multirow[t]{4}{*}{13} & -8 & 500 & 2000 & - & - \\
\hline & 0 & 500 & 2000 & - & - \\
\hline & +2 & 1000 & 2000 & - & + \\
\hline & +35 & 250 & 2000 & - & - \\
\hline 14 & 0 & 125 & 1000 & - & - \\
\hline 15 & 0 & 250 & 1000 & - & - \\
\hline \multirow[t]{3}{*}{16} & 0 & 125 & 1000 & - & + \\
\hline & +4 & 125 & 2000 & - & + \\
\hline & +8 & 250 & 2000 & - & + \\
\hline 17 & 0 & 32000 & 32000 & + & + \\
\hline \multirow[t]{2}{*}{18} & 0 & 4000 & NT & - & + \\
\hline & +4 & 1000 & NT & - & + \\
\hline \multirow[t]{2}{*}{19} & 0 & 8000 & 64000 & + & + \\
\hline & +12 & 4000 & 64000 & + & + \\
\hline \multirow[t]{4}{*}{20} & 0 & 250 & 128 & + & + \\
\hline & +1 & 1000 & 512 & + & + \\
\hline & +4 & 500 & 4000 & + & + \\
\hline & +16 & 500 & 2000 & + & + \\
\hline
\end{tabular}

* 0 , Time of presentation; NT, not tested; - , before clinical presentation; + , after clinical presentation.

+ , positive result; - , negative result. response in one case. Six patients had serological evidence of a primary infection with $T$. gondii; the presence of specific IgM was demonstrated by DSELISA and ISAGA. In 10 patients there was no demonstrable IgM response to cerebral infection. In two cases a low level of IgM (ISAGA positive, DSELISA negative) was noted at presentation and in the remaining two patients there was serological evidence of prior exposure to $T$. gondii but they mounted a low level IgM response in association with cerebral reactivation. Diagnostic brain biopsy was performed on two patients; in each case histology and nucleicacid detection indicated toxoplasma infection but the parasite was not isolated in tissue culture. Significant morbidity was not recorded in association with the operative procedures. The cerebrospinal fluid (CSF) was sampled by lumbar puncture in three patients and repeated in one case. Two patients had specific IgG but not IgM in the CSF accompanied by a raised protein level but normal glucose; there were no white blood cells and no micro-organisms were seen by microscopy or isolated by culture. The CSF sample from the remaining patient was unremarkable.

Complete records of management and clinical progression were obtained for 12 patients. Sulphonamide plus pyrimethamine was the initial therapy in 10 cases and one patient was given clindamycin plus pyrimethamine treatment, having had a severe sulphonamide reaction during earlier treatment of pneumocystis pneumonia. Of the 10 patients receiving sulphonamides, four suffered a significant adverse reaction and on two occasions these were sufficiently severe to warrant a change of treatment; clindamycin plus pyrimethane was the alternative therapy in both cases. One patient received no specific treatment and died 20 days after presentation. Cerebral toxoplasmosis was demonstrated at post-mortem examination. All 11 patients given specific therapy showed a marked clinical response within 2 weeks of commencing treatment and were alive 6 weeks after initial presentation. Resolution of $\mathrm{CT}$ abnormalities was documented in five cases. Reduced dose maintenance therapy was given to all treated patients on discharge from hospital - sulphonamide plus pyrimethamine in eight cases, clindamycin plus pyrimethamine in one case and dapsone for two individuals. These 11 cases were followed-up for 2-10 months and no relapses of cerebral toxoplasma infection were recorded.

\section{Discussion}

The definitive diagnosis of cerebral toxoplasmosis in association with AIDS remains problematic. Histological samples are rarely made available; consequently most cases are identified on the basis of compatible clinical, radiological and serological findings and the diagnosis is "confirmed" by clinical and radiological response to specific therapy. ${ }^{12}$ The conventional approach to the diagnosis of cerebral 
toxoplasmosis will bias any study against the recognition of cases presenting atypically. The potential toxicity of anti-parasitic drugs limits the application of specific therapy as a diagnostic tool and lack of response to these agents is likely to be ascribed to misdiagnosis rather than treatment failure. Studies from North America indicate the preponderance, in patients with AIDS, of cases of cerebral toxoplasmosis associated with secondary reactivation of parasitic infection compared with instances of primary disease. ${ }^{4}$ Serological parameters are well defined in cases of primary infection but remain uncertain in the diagnosis of reactivated disease. In practice, the diagnosis of primary infection (associated with seroconversion, rising antibody titres and specific IgM production) can be made with greater confidence than that of secondary reactivation of infection, and the current study may over-emphasise the incidence of primary infection. The cases identified were predominantly amongst male homosexuals and the findings should be applied with caution to other major groups of AIDS patients, such as drug abusers and haemophiliacs. Detailed studies of these groups are required to exclude significant differences in the presentation of cerebral toxoplasmosis. False positive and false negative findings have been recorded with most tests used for the serological investigation of suspected toxoplasmosis. ${ }^{713}$ The use of multiple assays for the measurement of IgG and IgM, including the dye test, the reference method for $T$. gondii antibody studies, will have minimised error due to technical limitations.

The clinical findings noted at presentation and the results of $\mathrm{CT}$ examination were similar to those reported in studies from the USA. ${ }^{14,15}$ Contrast studies were employed irregularly in the present assessment, reflecting the different practices of the reporting centres, but contrast injection was of diagnostic value in all cases in which it was used. CSF was sampled in only a minority of cases. The presence of space-occupying lesions exerting mass effect was stated to be the commonest contra-indication to lumbar puncture. One American group has promoted the diagnostic use of CSF sampling ${ }^{16}$ and the different practice observed in the present study may reflect the availability of facilities for ventricular canulation. Mortality and morbidity associated with sampling of CSF in AIDS patients has not been studied in detail. When CSF samples were obtained in the current study, the results did not contribute to the eventual diagnosis.

\section{References}

1. Ruskin J, Remington JS. Toxoplasmosis in the compromised host. Ann Intern Med 1976; 84: 193-199.

2. Carter AO, Frank JW. Congenital toxoplasmosis: epidemiologic features and control. Can Med Assoc J 1986; 135: 618-623.

3. Wreghitt TG, Hakim M, Gray JJ et al. Toxoplasmosis in heart and heart and lung transplant recipients. $J$ Clin Pathol $1989 ; 42: 194-199$.
The detection of specific antibody in the peripheral blood of all AIDS patients with cerebral toxoplasmosis is well established and negative findings exclude the diagnosis. ${ }^{17}$ The quantitative assessment of $\mathrm{IgG}$ showed marked case-to-case variation and antibody levels should not be used as a diagnostic criterion. Whereas previous studies have failed to demonstrate specific IgM in the majority of cases, IgM was detected in half of the present study group. The reliable diagnosis of primary infection will lead to the consistent identification of such patients but the difficulties of diagnosis in cases of secondary reactivation may have resulted in the failure to make a definitive decision and the exclusion of some cases of cerebral toxoplasmosis from this study. It is notable that of the 10 cases in which IgM was detected, four gave positive reactions by ISAGA and not by DSELISA. ISAGA has been shown to be more sensitive than DS-ELISA ${ }^{9}$ and to be of greater diagnostic value when investigating samples of fetal or neonatal serum. ${ }^{18}$ The results reported here indicate that ISAGA may be the assay of choice for the measurement of toxoplasma specific IgM in AIDS patients with cerebral disease. In cases 12 and 13 the patients had specific antibody in samples taken before the episode of cerebral disease but produced IgM detectable by ISAGA only with the onset of secondary reactivation. Some cases of cerebral toxoplasmosis in AIDS patients associated with secondary reactivation of infection may be accompanied by low level specific IgM production which can be detected by a highly sensitive assay. If confirmed, this would be a valuable diagnostic finding and further studies of this aspect are required.

Treatment prescribed by clinicians in the UK reflects the experience and recommendations of their North American colleagues. As in the USA, sulphonamide plus pyrimethamine is regarded as first line therapy although the high incidence of toxicity necessitates the administration of alternative regimens in a proportion of cases. There is an urgent need for the introduction of novel, effective agents for the prophylaxis, acute therapy and maintenance treatment of toxoplasmosis in patients with AIDS.

The development of a system for $T$. gondii specific nucleic acid detection by use of PCR was supported by a grant from the Medical Research Council. I am grateful to Mrs J. D. Johnson for technical assistance and to Mrs I. Crossman for preparation of the typescript.

4. Luft BJ, Remington JS. Toxoplasmic encephalitis. J Infect Dis $1988 ; 157 ; 1-6$

5. Gransden WR, Brown PM. Pneumocystic pneumonia and disseminated toxoplasmosis in a male homosexual. $\mathrm{Br} \mathrm{Med}$ $J$ 1983; 286 : 1614.

6. Sabin AB, Feldman HA. Dyes as microchemical indicators of a new immunity phenomenon affecting a protozoon parasite (Toxoplasma). Science 1948; 108: 660-663.

7. Holliman RE, Johnson J, Duffy K, New L. Discrepant toxoplasma latex agglutination test results. $J$ Clin Pathol $1989 ; 42:$ 200-203. 
8. Payne RA, Joynson DHM, Balfour AH et al. Public Health Laboratory Service enzyme linked immunosorbent assay for detecting Toxoplasma specific IgM antibody. J Clin Pathol 1987; 40: 276-281.

9. Duffy KT, Wharton PJ, Johnson JD, New L, Holliman RE Assessment of an immunoglobulin-M immunosorbent agglutination assay (ISAGA) for detecting toxoplasma specific IgM. J Clin Pathol 1989; 42: 1291-1295.

10. Savva D, Morris JC, Johnson JD, Holliman RE. Polymerase chain reaction for detection of Toxoplasma gondii. $\mathrm{J} \mathrm{Med}$ Microbiol 1990; 32: 25-31.

11. Hughes HPA, Hudson L, Fleck DG. In vitro culture of Toxoplasma gondii in primary and established cell lines. Int $J$ Parasitol 1986; 16 : 317-322.

12. Holliman RE. Toxoplasmosis and the acquired immune deficiency syndrome. $J$ Infect $1988 ; 16: 121-128$.

13. Naot Y, Luft BJ, Remington JS. False-positive serological tests in heart transplant recipients. Lancet 1981 ; 2: 590-591.
14. The TE Study Group. Haverkos HW. Assessment of therapy for toxoplasma encephalitis. Am J Med 1987; 82: 907-914.

15. Elkin CM, Leon E, Grenell SL, Leeds NE. Intracranial lesions in the acquired immunodeficiency syndrome. JAMA 1985; 253: 393-396.

16. Potsman I, Resnick L, Luft BJ, Remington JS. Intrathecal production of antibodies against Toxoplasma gondii in patients with toxoplasmic encephalitis and the acquired immunodeficiency syndrome (AIDS). Ann Intern Med 1988; 108: 49-51.

17. Navia BA, Petito CK, Gold JW, Cho ES, Jordan BD, Price RW. Cerebral toxoplasmosis complicating the acquired immune deficiency syndrome: clinical and neuropathological findings in 27 patients. Ann Neurol 1986; 19: 224-238.

18. Holliman RE, Johnson JD. The post-natal serodiagnosis of congenital toxoplasmosis. Serodiag Immunother 1989; 3: 323-327. 